Note

\title{
Effect of Vitamin E and C Supplements on Antioxidant Defense System in Cardiovascular Disease Patients in Zahedan, Southeast Iran
}

\author{
Mansour KARAJIBANI ${ }^{1}$, Mohammad HASHEMI ${ }^{2, *}$, Farzaneh MONTAZERIFAR ${ }^{1}$ and \\ Madhurima DIKSHIT ${ }^{3}$ \\ ${ }^{1}$ Department of Nutrition, and ${ }^{2}$ Department of Clinical Biochemistry, School of Medicine, \\ Zahedan University of Medical Sciences, Zahedan, Iran \\ ${ }^{3}$ Department of Chemistry, Division of Biochemistry, Pune University, Pune-411007, India
}

(Received April 26, 2010)

\begin{abstract}
Summary Oxidative stress plays an important role in the pathogenesis of cardiovascular disease (CVD). Growing evidence suggest that antioxidant vitamins might reduce the risk of disease outcomes by their ability to scavenge free radicals. The aim of the present study was to evaluate the supplementation of vitamins $\mathrm{E}$ and $\mathrm{C}$ on oxidant and antioxidant status in CVD patients. We conducted a case-control study with vitamin E (400 IU/d) and vitamin C $(500 \mathrm{mg} / \mathrm{d}$ ) supplementation in $40 \mathrm{CVD}$ patients for $2 \mathrm{mo}$. Antioxidant (enzymatic and nonenzymatic) and oxidant status were analyzed pre and post supplementation. In the initial stage the activity of both enzymatic and non-enzymatic antioxidants were lower, while the malondialdehyde (MDA) level was elevated $(p<0.0001)$. After intervention, a significant increase in superoxide dismutase (SOD) activity $(61.7 \%)$, glutathione peroxidase (GPx) activity $(59.3 \%)$, the levels of vitamin E (83.7\%), C (145.3\%), total antioxidant capacity (TAC) $(62.8 \%)$ and a significant decrease in MDA $(40 \%)$ value were observed $(p<0.0001)$. There was a significant negative correlation between MDA and TAC. The results suggest that supplementation with a combination of vitamins $\mathrm{E}$ and $\mathrm{C}$ reduced lipid peroxidation and strengthened the antioxidant defense system. Hence, there will be beneficial effects on the heart by reducing oxidative stress in CVD patients.
\end{abstract}

Key Words vitamin E and C supplementation, lipid peroxidation, cardiovascular disease (CVD)

Oxidative stress plays an important role in the pathogenesis of cardiovascular disease (CVD). Numerous studies over the past decade have focused on the role of oxidative stress in $\operatorname{CVD}(1,2)$. The effect on cardiovascular function may occur in two ways; either in the longterm development of atherosclerosis or in immediate damage during a heart attack or stroke (3). Both enzymatic - such as superoxide dismutase (SOD), catalase, and glutathione peroxidase (GPx) - and non-enzymatic antioxidants strengthen the system of the body. Lipid peroxidation, oxidation of low density lipoprotein (LDL) in particular, is the main causative factor in the development of CVD (4). During oxidation, peroxides followed by proxy radicals are formed which further degrade to aldehydes; malondialdehyde (MDA), hexanal and 4-hydroxynonenal (5). Antioxidant nutrients such as vitamins $\mathrm{C}$ and $\mathrm{E}$ are capable of inhibiting lipid peroxidation and reducing cardiovascular events $(1,6,7)$. There is substantial evidence that free radicals affect the etiology of many diseases including vascular disease (8). At the same time the living organisms have antiox-

\footnotetext{
*To whom correspondence should be addressed.

E-mail:mhd.hashemi@gmail.com
}

idant defense systems which prevent the damaging effects of excessive endogenously and exogenously produced free radicals (9). Non-enzymatic detoxification against reactive oxgen species (ROS) is determined by lipid-soluble antioxidants including vitamin $\mathrm{E}$ and carotenoids, and vitamin $\mathrm{C}$, a water-soluble antioxidant present in the extracellular fluid of the arterial wall (10-12). Observational studies have concluded that high intakes of antioxidant vitamins such as vitamin $\mathrm{E}$ and $\mathrm{C}$ from food and supplements are related to low risk of $\operatorname{CVD}(13,14)$. Some clinical trials have reported conflicting conclusions regarding the benefit of antioxidant vitamin supplement, which have not been confirmed $(7,9)$. Some studies have shown that mild to moderate deficiencies of vitamins $\mathrm{E}$ and $\mathrm{C}$ cause classic deficiency diseases, which may be involved in the development of CVD (11). So it is thought that antioxidant supplementation may help reduce the progression of CVD (1). Individuals with high dietary intakes of vitamin $\mathrm{C}, \mathrm{E}$ and $\beta$ carotene have a lower risk of CAD (12). Many of the ingredients in commercial supplement packs have been demonstrated singly to have direct or indirect effect on the cellular antioxidant system (13).

Overall reported studies have shown inconsistent 
Table 1. Demographic and clinical characteristics of the study groups.

Groups

\begin{tabular}{lccc}
\multicolumn{1}{c}{ Parameters } & \multicolumn{2}{c}{ CVD patients } & Control group $(n=63)$ \\
\cline { 2 - 3 } & Before intervention $(n=40)$ & After intervention $(n=31)$ & \\
\hline Age $(\mathrm{y})$ & $57.7 \pm 10.6$ & - & $56.4 \pm 11.3$ \\
Sex $(\mathrm{F} / \mathrm{M})$ & $28 / 12$ & $23 / 8$ & $41 / 22$ \\
BMI $\left(\mathrm{kg} / \mathrm{m}^{2}\right)$ & $27.4 \pm 4.9$ & $26.9 \pm 4.8$ & $26.5 \pm 4.6$ \\
Blood pressure $(\mathrm{mmHg})$ & $146.3 \pm 17.2$ & $125.3 \pm 17$ & $113.1 \pm 12.7$ \\
$\quad$ Systolic & $93.3 \pm 12.6$ & $78.9 \pm 11.6$ & $72.6 \pm 9.4$ \\
$\quad$ Diastolic & & \\
\hline
\end{tabular}

results: beneficial effect of individual antioxidants, combined effect and no effect of vitamin $\mathrm{E}$ and $\mathrm{C}\left(1,3,8_{-}\right.$ 10). Thus, the current evidence is insufficient to conclude that antioxidant vitamin supplementation reduces oxidative damage in humans. It will be important to clarify the discrepancy between the results of different studies. The aim of this study was to examine the combined effect of supplementation of vitamins $\mathrm{E}$ and $\mathrm{C}$ on oxidant and antioxidant defense systems in Iranian CVD patients.

\section{Materials and Methods}

Subject characteristics. Forty patients with CVD $(57.7 \pm 10.6 \mathrm{y})$ were enrolled in the study. The diagnosis of CVD patients, admitted to the intensive cardiac care unit (CCU and Post-CCU) wards at Khatam-Al-Anbia Hospital of Zahedan University of Medical Sciences, was established according to clinical criteria (unstable angina, and chest pain which lasted for up to $3 \mathrm{~h}$ ), echocardiography changes and total creatine kinase (CK) and $\mathrm{CK}_{\mathrm{MB}}$ activity.

Sixty-three healthy subjects $(56.4 \pm 11.3 \mathrm{y})$ were selected as the control group. They did not have any history of disease and were not on any medication. None of the study subjects received vitamin supplements in the 3 mo prior to the study. The protocol of the study was approved by the local ethical committee of Zahedan University of Medical Sciences and informed consent was obtained from all patients and healthy subjects.

Interventional study. The vitamin antioxidant supplementation was carried out by supplying both vitamin C (500 mg L-ascorbic acid, Darou Pakhsh Pharmaceutical Mfg. Co, Tehran, Iran) and vitamin E (400 IU, $d l-\alpha$-tocopheryl acetate, manufactured by international agencies for Health Burst, Davie, FL, USA). The patients were instructed to consume vitamin $\mathrm{C}$ and vitamin $\mathrm{E}$ daily for the duration of 2 mo. After 2 mo of supplementation, the effect of supplements was assessed by measurement of oxidant and antioxidants markers in the patients.

Blood sampling and analysis. Following a 12-h fast period, venous blood samples were collected in different tubes, before and after intervention and once for the control group. Heparinized tubes were used for mea- surement of MDA, total antioxidant capacity (TAC), SOD, GPx, and vitamin $\mathrm{C}$ and tubes without anticoagulant were used for the measurement of vitamin $\mathrm{E}$. Plasma and serum were obtained by centrifugation at $3,000 \mathrm{rpm}$ for $10 \mathrm{~min}$ and stored at $-80^{\circ} \mathrm{C}$ until analyzed.

MDA assay. Plasma MDA level was measured based on the reaction between MDA and thiobarbituric acid (TBA) using the method described by Satoh (14). The absorbance was read at $530 \mathrm{~nm}$ and expressed as micromoles of malondialdehyde per liter.

TAC. Total antioxidant capacity of plasma was measured by the ferric reducing ability of plasma (FRAP) assay. At low $\mathrm{pH}$, reduction of ferric-tripyridyltriazine complex to the ferrous form displays an intense blue color with an absorption maximum at $593 \mathrm{~nm}$ (15).

SOD and GPx activities. SOD and GPx were measured using commercially available kits supplied by Randox Laboratories Ltd (Randox, UK) as described previously (16).

Vitamins E and C assay. Serum levels of vitamin E were determined using high pressure liquid chromatography (HPLC) as described previously (16). Plasma concentration of vitamin $\mathrm{C}$ was measured by a spectrophotometric method (17) as described previously (16).

Statistical analysis. Results are expressed as means \pm SD. Statistical analysis was performed using SPSS 17 software (SPSS Inc, Chicago, USA). Paired and unpaired sample $t$-tests were used to compare the groups. $p$-values less than 0.05 were considered statistically significant.

\section{Results}

The demographic and clinical characteristics of CVD patients and healthy subjects are shown in Table 1. During the experimental period, 9 patients withdrew due to open heart surgery or transferred to another city for continuing treatment.

The levels of vitamin C, vitamin E, SOD, GPx, MDA, and TAC among normal subjects and CVD patients (before and after 2 mo supplementation) are shown in Fig. 1.

After 2 mo of supplementation, plasma level of vitamin C increased significantly (almost 1.5 fold) $(p<0.0001)$ from baseline and reached the normal level 
$1 \mathrm{~A}$

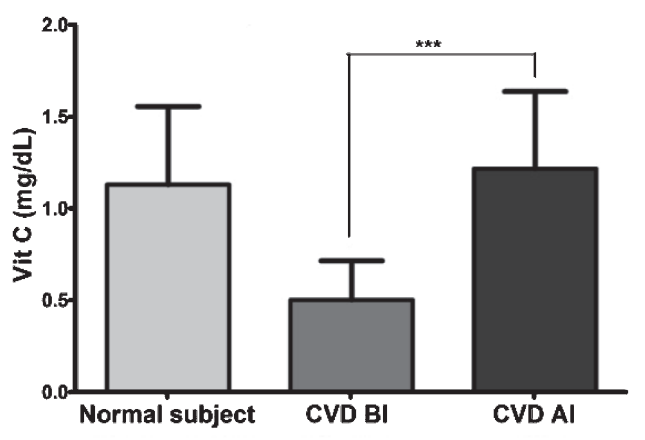

$1 \mathrm{C}$

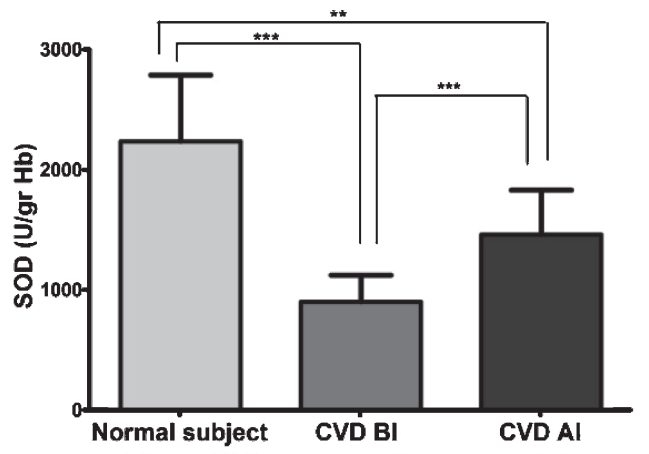

$1 E$

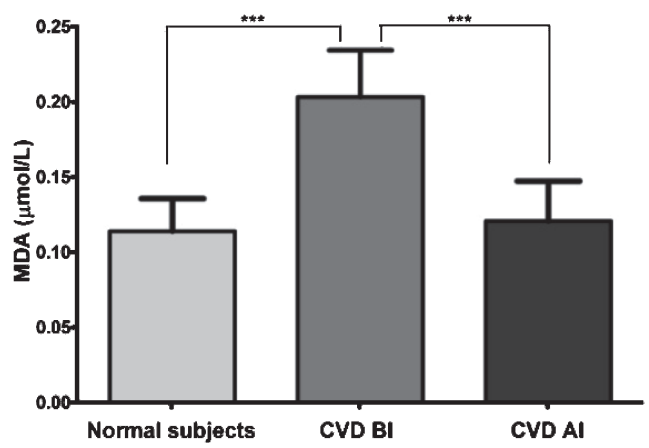

1B

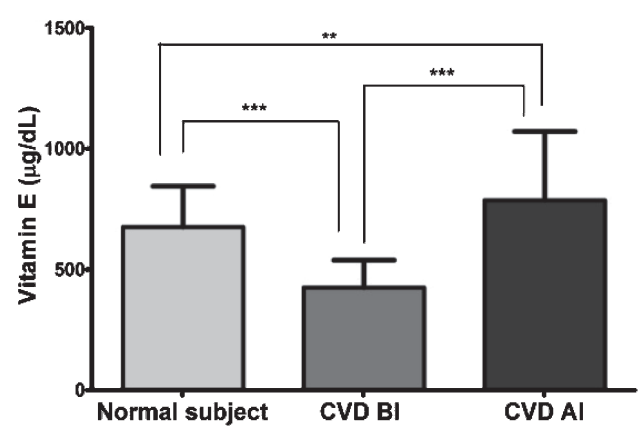

1D

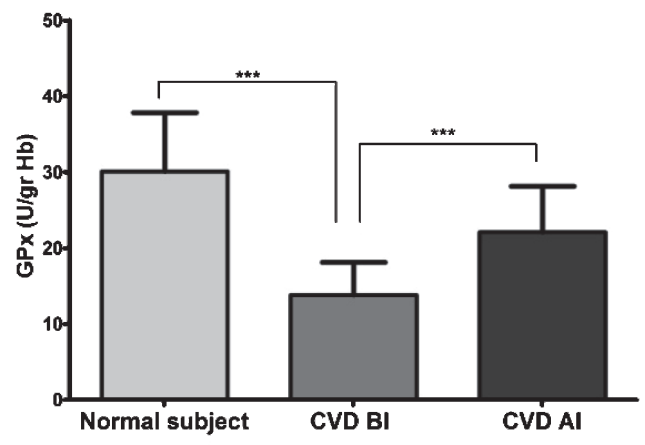

$1 F$

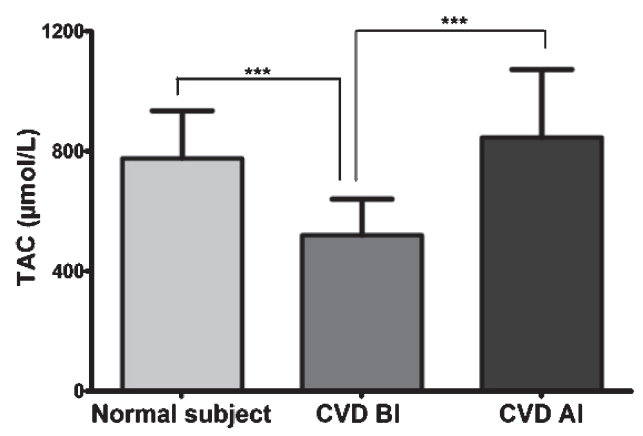

Fig. 1. Comparison the levels of vitamin C (A), vitamin E (B), SOD (C), GPx (D), MDA (E), and TAC (F) among normal subjects and cardiovascular patients before intervention (CVD BI) and after 2 mo supplementation (CVD AI) with vitamin $\mathrm{C}$ and vitamin E. ${ }^{* *} p<0.001,{ }^{* * *} p<0.0001$.

(Fig. 1A).

Similarly, the level of vitamin E increased significantly $(83.7 \%)(p<0.0001)$ so that it was higher than in normal subjects $(p<0.001)$ (Fig. 1B). Despite significant increase in the activities of SOD $(61.7 \%)$ and GPx $(59.3 \%)(p<0.0001)$, they were still significantly lower than for the control group $(p<0.001)$ (Fig. $1 \mathrm{C}$ and D). Plasma levels of MDA considerably decreased (40\%) $(p<0.0001)$ from baseline and reached the same level as the control value (Fig. 1E). Plasma total antioxidant capacity (TAC) after the 2-mo experimental period, increased significantly $(62.8 \%) \quad(p<0.0001)$ and reached that of the healthy individuals (Fig. 1F). A negative significant correlation was observed among MDA with GPx $(r=-0.37, p=0.04)$ and TAC $(r=-0.30$, $p=0.003)$ after intervention.

\section{Discussion}

The role of free radicals in the development of complications of CVD has been established by various epidemiological and clinical trials with antioxidants $(8,10,11$, 13). The increased level of MDA and lipid peroxidation products confirms the oxidation of LDL in CVD subjects (18). After 2 mo of vitamin $\mathrm{E}$ and $\mathrm{C}$ supplementation, MDA levels fell to almost normal values. Though vitamin C deficiency has been shown to be a risk factor for CHD $(7,9,11,19,20)$, some studies have indicated that there is an inverse association between CVD and high dietary vitamin $\mathrm{C}$ intakes or high plasma vitamin $\mathrm{C}$ concentrations, whereas other studies have not found any association between CVD and high plasma vitamin C concentration or use of supplements $(9,19)$. Vitamin $\mathrm{C}$ may enhance endothelial function by scavenging oxy- 
gen-derived free radicals, including superoxide anions, which interact with nitric oxide and impair its vasoactive function (4). Experimental studies have shown that lipid peroxidation is detectable only after all ascorbate has been completely used up $(9,19)$. However, vitamin $\mathrm{C}$ inhibition of lipid peroxidation in vivo has not yet been confirmed in supplementation studies in humans (19). Yet, clinical trials suggested vitamin $\mathrm{E}$ to be a more potential antioxidant $(1,2,7)$. In vitro studies have shown that LDL oxidation does not occur until vitamin E has been oxidized (21). In the presence of vitamin E, LDL has been shown to resist oxidation both in vivo (2, $7,8,22)$ and in vitro $(2,8,21,22)$. Besides, vitamin $\mathrm{E}$ decrease the cytotoxicity of oxidized LDL in the endothelial cells (7). Although it has been shown that treatment with a high dose of tocopherol reduces the risk of MI and CVD death in patients with ischemic heart disease (IHD), the other studies heve reported that vitamin E had no significant effect on nonfatal MI, stroke or death (22). Significantly lower plasma concentrations of vitamin $\mathrm{E}$ have been found in patients with angina pectoris than in normal subjects, and reduced levels of vitamin $\mathrm{C}$ in the leukocytes are predictive of evidence for CAD (7). Vitamin $\mathrm{C}$ is also important for maintaining levels of antioxidant vitamin $\mathrm{E}$ by reducing the vitamin E radical. Each antioxidant nutrient has specific activities. Yet in combination they are found to be more effective, suggesting synergistic action (3, 10). During oxidation, lipid radicals generated are scavenged by vitamin $\mathrm{E}$ and in the process get transformed to radicals which are recovered by vitamin $C(2)$, thus helping to maintain the concentration of vitamin $\mathrm{E}(1,2)$. Vitamin E seems to have the most cardio-protective potential (2). Both enzymatic (SOD and GPx) and non-enzymatic antioxidant vitamins (E and $\mathrm{C}$ ) and TAC low levels in CVD patients point towards their weak defense system which is strengthened by supplementation of antioxidants, and concentrations increase after $2 \mathrm{mo}$, as compared to controls. Similarly, several studies have reported that GSH levels as well as SOD activity were significantly decreased in CVD patients $(4,23)$ except that of McMurray et al. (24). The antioxidant system can be strengthened directly or indirectly by supplying antioxidants $(12,13)$. It is reported that the combination of vitamin $\mathrm{E}$ and $\mathrm{C}$ as antioxidant supplementation is safe in atherosclerosis prevention (25). The preventive effect of the vitamin antioxidant supplement depends on various factors: dose, singly and or in combination, duration and disease stage of administration, along with lifestyle $(8,10)$. It is reported that a high dose of supplementation (800 IU of vitamin E, $1,000 \mathrm{mg}$ of vitamin $\mathrm{C}$, and $24 \mathrm{mg}$ of $\beta$-carotene) for a period of $12 \mathrm{wk}$ significantly reduced the susceptibility of LDL to oxidation, whereas a mild dose (400 IU of vitamin $\mathrm{E}, 500 \mathrm{mg}$ of vitamin $\mathrm{C}$, and $12 \mathrm{mg}$ of $\beta$-carotene) did not have any effect (26). However, although supplementation with the combination dose of 400 IU $\alpha$-tocopherol acetate and $500 \mathrm{mg}$ ascorbic acid was tried for a period of $2 \mathrm{mo}$ in the present study, our results showed that supplementation of the combination of vitamins $\mathrm{E}$ and $\mathrm{C}$ reduced lipid peroxidation, and both vitamins acted as free-radical scavengers. These vitamins also strengthened the antioxidant defense system against oxidative damage, which was confirmed by the inverse correlation among MDA with GPx and TAC. Hence, supplementation of vitamins $E$ and $C$ may have beneficial effects in CVD patients which remain to be clarified.

\section{Acknowledgments}

The authors would like to thank Dr. Ahmad Bolouri (Cardiologist), the experts of the laboratory of National Nutrition and Food Technology Research Institute, Iran and the CCU/Post-CCU section staff in Zahedan Khatam-Al-Anbia Hospital for their kind cooperation and also the patients and healthy subjects who willingly participated in the study.

Conflict of Interests: None.

\section{REFERENCES}

1) Hasty AH, Gruen ML, Terry ES, Surmi BK, Atkinson RD, Gao L, Morrow JD. 2007. Effects of vitamin E on oxidative stress and atherosclerosis in an obese hyperlipidemic mouse model. J Nutr Biochem 18: 127-133.

2) Nuttall SL, Kendall MJ, Martin U. 1999. Antioxidant therapy for the prevention of cardiovascular disease. QJM 92: 239-244.

3) Bagchi K, Puri S. 1998. Free radicals and antioxidants in health and disease. East Mediterr Health J 4: 350360.

4) Serdar Z, Aslan K, Dirican M, Sarandol E, Yesilbursa D, Serdar A. 2006. Lipid and protein oxidation and antioxidant status in patients with angiographically proven coronary artery disease. Clin Biochem 39: 794-803.

5) Aviram M. 2003. Lipid peroxidation and atherosclerosis: the importance of selected patient group analysis. Isr Med Assoc J 5: 734-735.

6) Meagher E, Rader DJ. 2001. Antioxidant therapy and atherosclerosis: animal and human studies. Trends Cardiovasc Med 11: 162-165.

7) Diaz MN, Frei B, Vita JA, Keaney JF Jr. 1997. Antioxidants and atherosclerotic heart disease. $N$ Engl J Med 337: 408-416.

8) Duthie GG, Bellizzi MC. 1999. Effects of antioxidants on vascular health. Br Med Bull 55: 568-577.

9) Klipstein-Grobusch K, Geleijnse JM, den Breeijen JH, Boeing H, Hofman A, Grobbee DE, Witteman JC. 1999. Dietary antioxidants and risk of myocardial infarction in the elderly: the Rotterdam Study. Am J Clin Nutr 69: 261-266.

10) Jha P, Flather M, Lonn E, Farkouh M, Yusuf S. 1995. The antioxidant vitamins and cardiovascular disease. A critical review of epidemiologic and clinical trial data. Ann Intern Med 123: 860-872.

11) Morris CD, Carson S. 2003. Routine vitamin supplementation to prevent cardiovascular disease: a summary of the evidence for the U.S. Preventive Services Task Force. Ann Intern Med 139: 56-70.

12) Papas AM. 1999. Antioxidant Status, Diet, Nutrition, and Health, 1st ed, p 114-115, 464-466. CRC Press, Florida.

13) Cheng T, Zhu Z, Masuda S, Morcos NC. 2001. Effects of multinutrient supplementation on antioxidant defense 
systems in healthy human beings. I Nutr Biochem 12 : 388-395.

14) Satoh K. 1978. Serum lipid peroxide in cerebrovascular disorders determined by a new colorimetric method. Clin Chim Acta 90: 37-43.

15) Benzie IF, Strain JJ. 1996. The ferric reducing ability of plasma (FRAP) as a measure of "antioxidant power": the FRAP assay. Anal Biochem 239: 70-76.

16) Karajibani M, Hashemi M, Montazerifar F, Bolouri A, Dikshit M. 2009. The status of glutathione peroxidase, superoxide dismutase, vitamins A, C, E and malondialdehyde in patients with cardiovascular disease in Zahedan, Southeast Iran. J Nutr Sci Vitaminol 55: 309316.

17) Burtis CA, Ashwood ER. 2006. Tietz Textbook of Clinical Chemistry and Molecular Diagnostics, 4th ed, p 1023-1025. Elsevier Saunders, Missouri.

18) Nagyova A, Krajcovicova-Kudlackova M, Horska A, Smolkova B, Blazicek P, Raslova K, Collins A, Dusinska M. 2004. Lipid peroxidation in men after dietary supplementation with a mixture of antioxidant nutrients. Bratisl Lek Listy 105: 277-280.

19) Nyyssonen K, Parviainen MT, Salonen R, Tuomilehto J, Salonen JT. 1997. Vitamin C deficiency and risk of myocardial infarction: prospective population study of men from eastern Finland. BMJ 314: 634-638.

20) Singh RB, Ghosh S, Niaz MA, Singh R, Beegum R, Chibo H, Shoumin Z, Postiglione A. 1995. Dietary intake, plasma levels of antioxidant vitamins, and oxidative stress in relation to coronary artery disease in elderly subjects. Am J Cardiol 76: 1233-1238.

21) Reaven PD, Khouw A, Beltz WF, Parthasarathy S, Witztum JL. 1993. Effect of dietary antioxidant combinations in humans. Protection of LDL by vitamin E but not by beta-carotene. Arterioscler Thromb 13: 590-600.

22) Dutta A, Dutta SK. 2003. Vitamin E and its role in the prevention of atherosclerosis and carcinogenesis: a review. J Am Coll Nutr 22: 258-268.

23) Yegin A, Yegin H, Aliciguzel Y, Deger N, Semiz E. 1997. Erythrocyte selenium-glutathione peroxidase activity is lower in patients with coronary atherosclerosis. Jpn Heart J 38: 793-798.

24) McMurray J, Chopra M, Abdullah I, Smith WE, Dargie HJ. 1993. Evidence of oxidative stress in chronic heart failure in humans. Eur Heart J 14: 1493-1498.

25) Hathcock JN, Azzi A, Blumberg J, Bray T, Dickinson A, Frei B, Jialal I, Johnston CS, Kelly FJ, Kraemer K, Packer L, Parthasarathy S, Sies H, Traber MG. 2005. Vitamins $\mathrm{E}$ and $\mathrm{C}$ are safe across a broad range of intakes. Am J Clin Nutr 81: 736-745. 